\title{
Flow Diverter Timing Error Modeling and Optimization
}

\author{
Luo Dezhang ${ }^{1, a}$, Liu Guixiong ${ }^{1, b^{*}}$, Chen Guoyu ${ }^{1, c}$, Pan Yunfei ${ }^{2, d}$ \\ ${ }^{1}$ School of Mechanical and Automotive Engineering, South China University of Technology \\ Guangzhou, China \\ ${ }^{2}$ Guangzhou Institute of Energy Testing, Guangzhou, China \\ e-mail: 'medezhangluo@hotmail.com, bmegxliu@scut.edu.cn, 'mechenguoyu@hotmail.com, \\ dpanyunfei@163.net
}

Keywords: flow diverter; diverting flow error; timing error

\begin{abstract}
Timing error is one of the most important accuracy affected factors of the flying-startstop liquid flow calibration facilities. However, the traditional timing error calculation model has omitted several quadratic terms so it has some model errors. In this paper, a unified and complete model of timing error has been developed by analyzing the transforming relationship between diverting flow error and timing error, and, the new model can be use in the performance evaluation of different types of diverters. The experiments results reveal that the new calculation model has a higher error measurement precision, and, for both the new and the traditional timing error calculation model: the timing error will increase with a higher flow rate; the performance of using a fixed nozzle is better than that of using a moving nozzle; the timing error can be reduced effectively by adjusting the trigger location.
\end{abstract}

\section{Introduction}

The flying-start-stop liquid flow calibration facilities based on gravimetric method are widely used for the calibration of different types of flowmeters. In these facilities, flow diverter represents a key accuracy determining functional unit that its uncertainty is mainly depended on the diverting flow error. However, diverting flow error is difficult to be obtained directly. So generally, the timing error recommended by ISO4185 [1] and GB/T 17612-1998 [2] is used to estimate diverting flow error. Nevertheless, the traditional timing error model has omitted several quadratic terms so that it will cause some model errors, and, the traditional model is generally used in the facilities with a fixed nozzle [3-5]. Based on the related works mentioned above, this research proposed a transforming relationship model of diverting flow error and timing error to establish a complete timing error model which can be used in the performance evaluation of different types of flow diverters. At last, the performance comparing experiments of the new and the traditional timing error calculation model are proposed to verify the advantageous characteristics of the new model.

\section{Principle of Timing Error}

\subsection{Diverting Flow Error Model}

As shown in Fig. 1, a flow diverter is composed of a nozzle and a divider. The working principle of the flow diverter can be expressed as follows: 1) Before the measurement, diverter directs the liquid flow to storage tank; 2) Diverting in progress: At the beginning of the measurement, the divider/nozzle directs the liquid flow from storage tank to weighing tank at $t_{D I s t a r t}-t_{D I s t o p}$ which denote the diverting in progress starting and stopping timing respectively as shown in Fig. 1(b). Meanwhile, the trigger is activated and timing begins at $t_{\text {Mstart }}$; 3)Diverting out progress: After a constant flow rate collection interval, the divider/nozzle redirects the liquid flow from weighing tank to storage tank at $t_{D O s t a r t}-t_{D O s t o p}$ which denote the diverting out progress starting and stopping timing respectively. Meanwhile, the trigger is activated and timing stops at $t_{\text {Mstop }}$. 


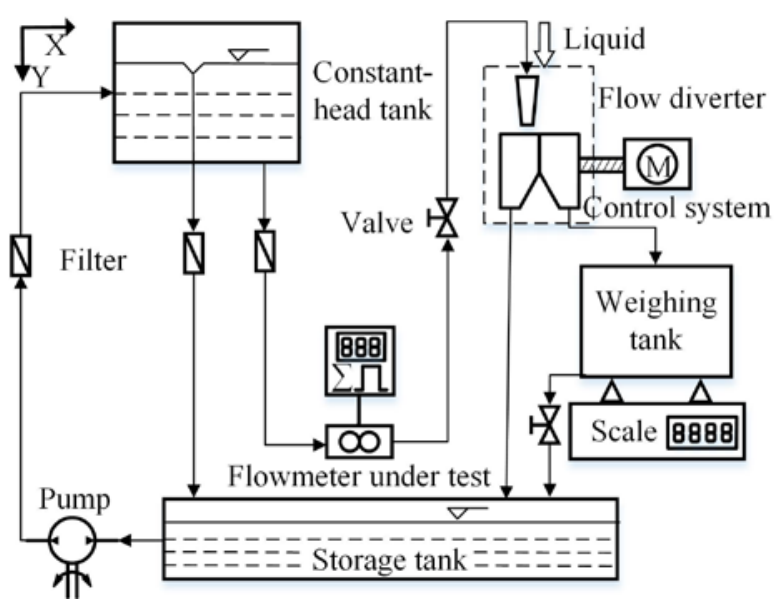

(a)

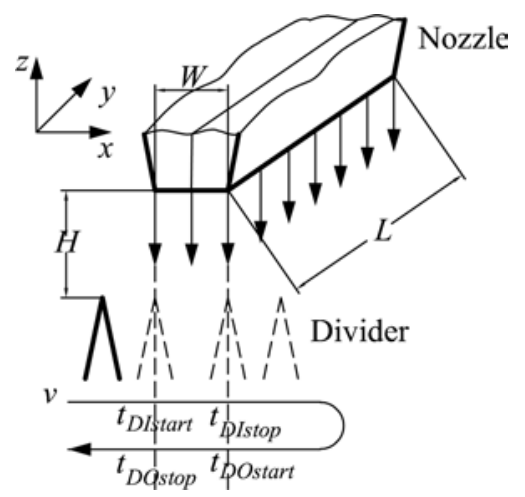

(b)

Fig. 1. Principle of the opposite directional diverter with a fixed nozzle. (a) Schematic of the gravimetric liquid flow calibration facility; (b) The motion law of the divider in diverting progress.

Finally, the mass flow rate $q_{C}$ used to calibrate the flowmeter under test can be given by (1) according to ISO4185:

$$
q_{C}=\frac{Q_{M W T}}{T_{M}}
$$

where, the $Q_{M w T}$ represents the liquid mass of weighing tank during the whole measurement, the $T_{M}$ represents the total measuring time and it is given by $T_{M}=t_{M s t o p}-t_{M s t a r t}$. However, improper timing starting and stopping points will cause the diverting flow error and lead to the error of the $q_{C}$.

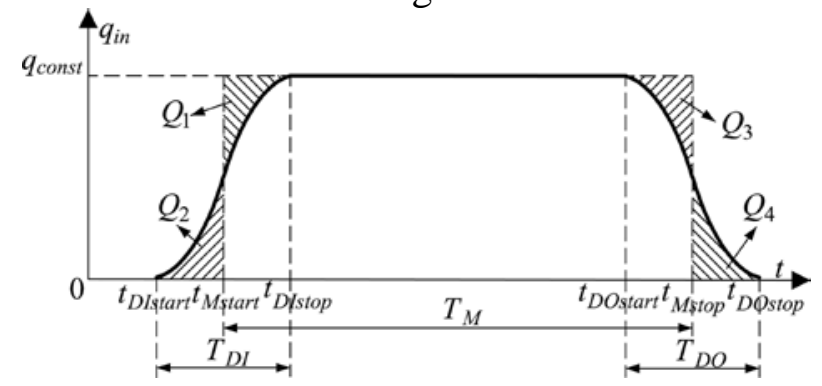

Fig. 2. The instantaneous flow rate model of weighing tank during the whole measurement.

As shown in Fig. 2, $T_{D I}$ and $T_{D O}$ denote the time interval of diverting in and diverting out progress, respectively. The $q_{\text {in }}$ and $q_{\text {const }}$ represent the changing and the constant flow rate directed to the weighing tank respectively. So the liquid mass of weighing tank during the whole measurement is $Q_{M W T}=\int_{t_{\text {DIsart }}}^{t_{\text {Dostop }}} q_{i n} d t$ and the liquid mass calculated by measuring time is $Q_{M C}=q_{\text {in }}\left(t_{M s t o p}-t_{M s t a r t}\right)$. Diverting flow error $Q_{M E}$ is given by (2):

$$
Q_{M E}=Q_{M W T}-Q_{M C}=Q_{2}-Q_{1}+Q_{4}-Q_{3}
$$

However, diverting flow error is very difficult to be obtained and eliminated directly. Though an attempt has been proposed to obtain diverting flow error by numerical system simulation [6]. However, it is a theoretical solution, and, it is unpractical and time-consuming to solve every different diverting flow error under a wide range of flow rates.

\subsection{Transforming Relationship between Diverting Flow Error and Timing Error}

To find out a physical index to estimate diverting flow error which is easier to be obtained, the concept of timing error is proposed.

As shown in Fig. 3. $q_{1}, q_{1 C}$, and $\Delta q_{1}$ denote the actual circuit flow rate, flow rate used to calibrate the flowmeter under test, and diverting flow rate error respectively; $m_{1}, m_{1 C}$ (gray region), and $\Delta m_{1}$ (diagonal region) denote the liquid mass of weighing tank, the liquid mass calculated by measuring time, and the diverting flow error respectively. $t^{\prime}{ }_{\text {Mstart }}$ and $t^{\prime}{ }_{\text {Mstop }}$ represent the actual timing starting and stopping point respectively. Let $t_{M s t a r t-O P}$ and $t_{M s t o p-O P}$ denote the optimal timing 
starting and stopping points respectively which make $Q_{M E}=0$. So the ideal timing interval is $t_{\text {ideal }}=t_{\text {Mstop-OP }}-t_{\text {Mstart-OP }}$, the actual timing interval is $t_{1}=t^{\prime}{ }_{M s t o p}-t^{\prime}{ }_{M s t a r t}$. So the liquid mass of weighing tank can be given by $m_{1}=q_{1} \cdot t_{\text {ideal }}$. The liquid mass calculated by actual measuring time is $m_{1 C}=q_{1} \cdot t_{1}$. And $m_{1}=m_{1 C}+\Delta m_{1}$ can be obtained according to (2). Translate $t_{1}$ along the $x$-axis to make $t_{M s t a r t-}$ ${ }_{O P}=t^{\prime}{ }_{M s t a r t}$. Then the relationship of $m_{1}, m_{1 C}$, and $\Delta m_{1}$ can be shown visually in Fig. 3 . Defined a new physical index called timing error which is given by $\Delta t=t_{\text {ideal }}-t_{1}$. The converting relationship of diverting flow error and timing error is given by:

And $q_{1}$ can be given by:

$$
\Delta m_{1}=\Delta q_{1} \bullet t_{1}=q_{1} \bullet \Delta t
$$

$$
q_{1}=\frac{m_{1}}{\left(t_{1}+\Delta t\right)}=\frac{m_{1}}{t_{1}} \cdot \frac{1}{1+\Delta t / t_{1}}
$$

To avoid losing significance digits of $\Delta t$ and eliminated the influence caused by the unsteady of $q_{1}$, the circuit flow rate is kept constant and the diverter performs one diversion in each equal time interval $t_{i}=t_{1} / n\left(n \in N^{+}, n \geq 10,1 \leq \mathrm{i} \leq n\right)$ without resetting the timer and the weighing scale. At last, the diverter system will accumulate $n \Delta t$. The transforming model of the diverting flow error and the timing error after $n$ diversions can be obtained as shown in Fig. 4.

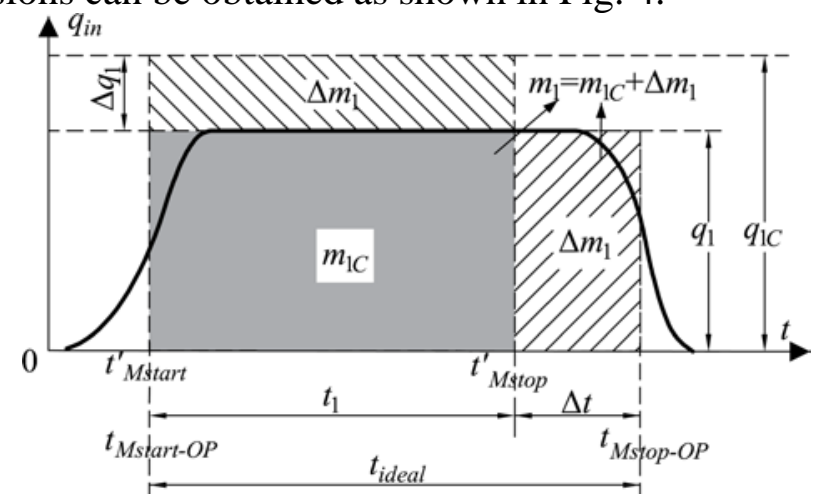

Fig. 3. The transforming relationship model of diverting flow error and timing error in the measurement with one diversion.

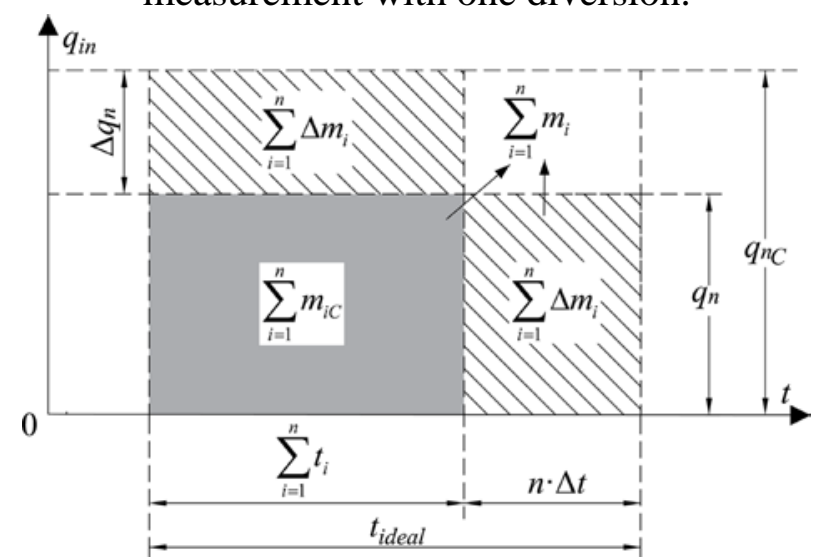

Fig. 4. The transforming relationship model of the diverting flow error and the timing error after $n$ diversions.

As shown in Fig. $4, q_{n}, q_{n c}$, and $\Delta q_{n}$ denote the actual circuit flow rate, flow rate used to calibrate the flowmeter, and diverting flow rate error respectively after $n$ diversions; $m_{i}, m_{i C}, \Delta m_{i}$, and $t_{i}$ denote the liquid mass of weighing tank, the liquid mass calculated by measuring time, the diverting flow error, and the timing interval in the ith diversion respectively. The $\sum_{i=1}^{n} m_{i}, \sum_{i=1}^{n} m_{i C}, \sum_{i=1}^{n} \Delta m_{i}$, and $\sum_{i=1}^{n} t_{i}$ denote the total liquid mass of weighing tank, the total liquid mass calculated by measuring time, the total diverting flow error, and the total measuring time respectively after $n$ diversions.

The $q_{n}$ can be given by: 


$$
q_{n}=\frac{\sum_{i=1}^{n} m_{i}}{\left(\sum_{i=1}^{n} t_{i}+n \bullet \Delta t\right)}=\frac{\sum_{i=1}^{n} m_{i}}{\sum_{i=1}^{n} t_{i}} \cdot \frac{1}{1+n \bullet \Delta t / \sum_{i=1}^{n} t_{i}}
$$

According to (4) and (5), the traditional calculation model of the timing error $\Delta t_{[1-2]}$ can be given by:

$$
\begin{gathered}
\frac{q_{n}}{q_{1}}=\frac{\sum_{i=1}^{n} m_{i} / \sum_{i=1}^{n} t_{i}}{m_{1} / t_{1}} \cdot \frac{1+\Delta t / t_{1}}{1+n \cdot \Delta t / \sum_{i=1}^{n} t_{i}} \\
\frac{\sum_{i=1}^{n} m_{i} / \sum_{i=1}^{n} t_{i}}{m_{1} / t_{1}} \cdot \frac{q_{1}}{q_{n}}=\frac{1+n \bullet \Delta t / \sum_{i=1}^{n} t_{i}}{1+\Delta t / t_{1}} \approx \frac{1+n \bullet \Delta t / t_{1}}{1+\Delta t / t_{1}} \\
=\frac{\left(1+n \bullet \Delta t / t_{1}\right)\left(1-\Delta t / t_{1}\right)}{1-\left(\Delta t / t_{1}\right)^{2}} \approx 1+(n-1) \Delta t / t_{1} \\
\Rightarrow \Delta t_{[1-2]}=\frac{t_{1}}{(n-1)}\left(\frac{q_{1}}{q_{n}} \frac{\sum_{i=1}^{n} m_{i} / \sum_{i=1}^{n} t_{i}}{m_{1} / t_{1}}-1\right)
\end{gathered}
$$

So the traditional timing error calculation model has omitted several quadratic terms $\left(\Delta t / t_{1}\right)^{2}$. The complete timing error calculation model $\Delta t$ can be given by:

$$
\left.\frac{\sum_{i=1}^{n} m_{i} / \sum_{i=1}^{n} t_{i}}{m_{1} / t_{1}} \cdot \frac{1+n \cdot \Delta t / \sum_{i=1}^{n} t_{i}}{q_{n}}-1=\frac{n \cdot \Delta t \cdot t_{1}-\Delta t \cdot \sum_{i=1}^{n} t_{i}}{1+\Delta t / t_{1}}-1=\frac{\sum_{i=1}^{n} t_{i}}{\sum_{i=1}^{n} t_{i}\left(t_{1}+\Delta t\right)} \Rightarrow \Delta t=\frac{\sum_{i=1}^{n} m_{i} / \sum_{i=1}^{n} t_{i}}{m_{1} / t_{1}} \cdot \frac{q_{1}}{q_{n}}-1\right)
$$

Let $k_{q}=q_{n} / q_{1} 、 k_{t}=\sum_{i=1}^{n} t_{i} / t_{1} 、 k_{m}=\sum_{i=1}^{n} m_{i} / m_{1}$, so,

$$
\left\{\begin{array}{l}
\Delta t_{[1-2]}=\frac{t_{1}}{(n-1)}\left(\frac{k_{m}}{k_{q} \bullet k_{t}}-1\right) \\
\Delta t=\frac{k_{t} \bullet t_{1}}{\left(n-k_{m} / k_{q}\right)}\left(\frac{k_{m}}{k_{q} \cdot k_{t}}-1\right)
\end{array}\right.
$$

According to (8) and $k_{t} \approx 1, k_{q} \approx 1$,

$$
\begin{aligned}
& \frac{\Delta t}{\Delta t_{[1-2]}}=\frac{k_{t}(n-1)}{\left(n-k_{m} / k_{q}\right)} \approx \frac{(n-1)}{\left(n-k_{m}\right)} \\
& \Rightarrow\left\{\begin{array}{l}
k_{m}>1, \Delta t>\Delta t_{[1-2]}>0 \\
k_{m}<1,0>\Delta t>\Delta t_{[1-2]}
\end{array}\right.
\end{aligned}
$$

Equation (9) reveals that the complete has a higher model precision that that of the traditional model.

The model mentioned above established the transforming relationship of diverting flow error and timing error. And the timing error can be used as a physical index to evaluate the performance of different types of flow diverters.

\section{Results and Discussion}

Fig. 5 shows the structure of the flow diverter with a moving nozzle (DMN) and the flow diverter with a fixed nozzle (DFN). The working fluid is water, the ambient temperature is $25^{\circ} \mathrm{C}$, the atmospheric pressure $1.01325 \times 10^{\wedge} 5 \mathrm{~Pa}$, the standard measurement time is $60 \mathrm{~s}$, the number of 
accumulated diversions $n=15$, the number of replicated experiments $N=10$.

The timing error results of the DMN and the DFN with the complete model and the traditional model under a instantaneous flow rate $50 \mathrm{~L} / \mathrm{min}, 150 \mathrm{~L} / \mathrm{min}, 250 \mathrm{~L} / \mathrm{min}, 350 \mathrm{~L} / \mathrm{min}, 450 \mathrm{~L} / \mathrm{min}$, 550L/min, 650L/min are shown in Fig. 6. The timing error adjusting experiment results of DMN and DFN are shown in Fig. 7.

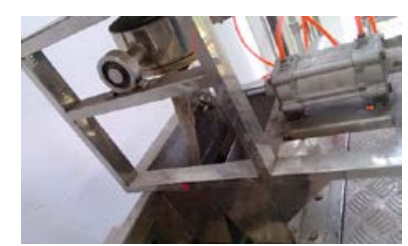

(a)DMN

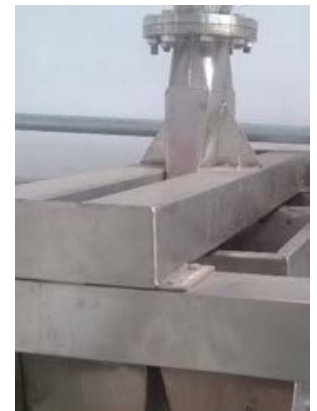

(b)DFN

Fig. 5 The structure of the flow diverter with a moving nozzle (DMN) and the flow diverter with a fixed nozzle (DFN).

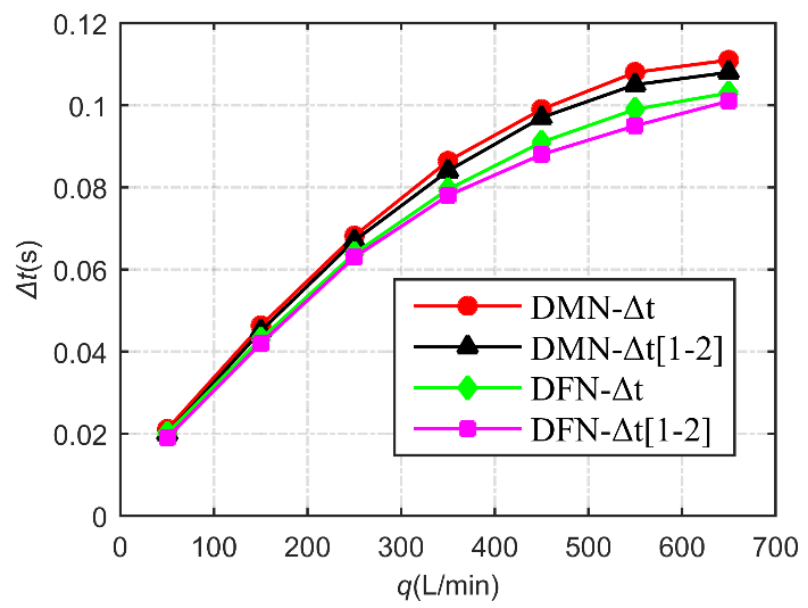

Fig. 6 The timing error results of the DMN and the DFN with the complete model and the traditional model.

As shown in Fig. 6, $\Delta t$ is the results of the complete model proposed in this paper, $\Delta t[1-2]$ is the results of the traditional model. According to Fig. 6 and Fig. 7, the results fit (9) that the new calculation model has a higher error measurement precision, and, for both the new and the traditional timing error calculation model: the timing error will increase with a higher flow rate; the performance of using a fixed nozzle is better than that of using a moving nozzle; the timing error can be reduced effectively by adjusting the trigger location.

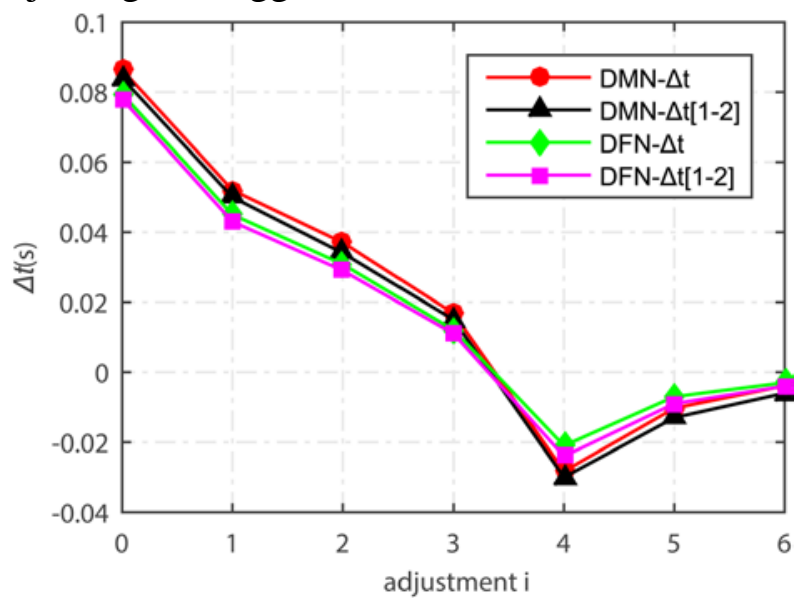

Fig.7 The timing error adjusting experiment results of DMN and DFN. 


\section{Conclusion}

This paper established the transforming relationship of diverting flow error and timing error and proposed a complete timing error calculation model that, the experiments results reveals that the new model has a higher error measurement precision than the traditional model. And the new model can be used as a physical index to evaluate the performance of different types of flow diverters.

\section{Acknowledgment}

This work was supported in part by the Science and Technology Planning Project of Administration of Quality and Technology Supervision of Guangzhou Municipality under Grant 2015KJ05, and, in part by the Science and Technology Planning Project of Guangdong Province under Grant 2016A040403044.

\section{References}

[1] Measurement of Liquid Flow in Closed Conduits-Weighing Methods, ISO 4185, 1980.

[2] Measurement of Liquid Flow in Closed Conduits-Weighing Methods, GB/T 17612-1998, 1998.

[3] T. Shimada, S. Oda, Y. Terao, and M. Takamoto, "Development of a New Diverter System for Liquid Flow Calibration Facilities,” Flow Meas. Instrum., Vol. 14, pp. 89-96, Feb, 2003.

[4] L. Cordova, T. Lederer, "A Practical Method to Assess the Performance of Gravimetric Flow Test Rigs by Using the Timing Error,” Flow Meas. Instrum., Vol. 44, pp. 61-70, Aug, 2015.

[5] T. Shimada, S. Oda, Y. Terao, M. Takamoto. Development of a new diverter system for liquid flow calibration facilities[J]. Flow Measurement and Instrumentation, 2003, 14(03): 89-96.

[6] R. Engel and H.J. Baade, "Model-Based Flow Diverter Analysis for an Improved Uncertainty Determination in Liquid Flow Calibration Facilities,” Meas. Sci. Technol., Vol. 21, pp. 1-11, Jan. 2010. 